\title{
Consumer opinions on adverse events associated with medicines and vaccines
}

This article was published in the following Dove Press journal:

Patient Preference and Adherence

\author{
Parisa Aslani' \\ Kim Hamrosi' \\ Vivien Tong' \\ Timothy F Chen' \\ Jane Cook ${ }^{2}$ \\ Romano Fois' \\ Theresa McGarry ${ }^{3}$ \\ Carter Moore ${ }^{4}$ \\ Rodney Peters ${ }^{5}$ \\ Sarah Spagnardi ${ }^{6}$ \\ Karen Whitelock ${ }^{7}$ \\ 'Faculty of Pharmacy, The University \\ of Sydney, Camperdown, NSW, \\ Australia; ${ }^{2}$ Therapeutic Goods \\ Administration, Canberra, \\ ACT, Australia; ${ }^{3}$ Celgene Pty \\ Ltd., Southbank, VIC, Australia; \\ ${ }^{4}$ Consumers Health Forum of \\ Australia, Canberra, ACT, Australia; \\ ${ }^{5}$ Pharmacovigilant Consultant, Sydney, \\ NSW, Australia; ${ }^{6}$ NPS MedicineWise, \\ Sydney, NSW, Australia; ${ }^{7}$ Novartis \\ Pharmaceuticals Australia Pty Ltd., \\ Sydney, NSW, Australia
}

Introduction: Despite the availability of an Australian consumer adverse event (AE) reporting system for over 50 years, reporting rates remain low. A comprehensive understanding of consumer perceptions and experiences regarding AEs is needed to further ascertain factors impacting their engagement in AE reporting.

Aim: The aim of this study was to explore consumer opinions about AEs potentially associated with medicines and vaccines, and their experiences and understanding of managing and reporting AEs.

Methods: Six focus groups were conducted across metropolitan Sydney with a total of 48 adult participants. A semi-structured focus group topic guide was developed to explore consumers' understanding, experiences, and actions taken in relation to AEs; and perspectives on managing treatment benefits and harms. Discussions were audio-recorded with participant permission and transcribed verbatim. Transcripts were thematically analyzed.

Results: Consumers acknowledged the potential for side effects (SEs), however inaccurately estimated SE risk in response to verbal descriptors such as "common." Consumer appraisal of treatment benefits and harms was influenced by factors such as medical condition(s), previous experiences, and beliefs. Although many had experienced SEs, consumers only reported them if considered severe or troublesome. Minimal awareness of consumer AE reporting systems was evident. Doctors were the primary avenue for reporting; consumers preferred doctors to act as the intermediary in reporting AEs to an independent body.

Conclusion: Consumers' lack of awareness of AE reporting systems was evident. With the complexities inherent in benefit/harm risk appraisal, information seeking, and AE reporting preferences, better consumer understanding of AEs and the systems available for reporting is needed.

Keywords: patient, reporting, side effects, qualitative, focus groups, drugs, vaccines

\section{Introduction}

All medicines and vaccines have the potential to lead to adverse events (AEs). An AE can be defined as "any untoward medical occurrence that may present during treatment with a pharmaceutical product but which does not necessarily have a causal relationship with this treatment". ${ }^{1}$ As clinical trials' data may not detect the incidence of AEs in the broader population, pharmacovigilance via postmarketing surveillance is used to monitor ongoing medication safety and any subsequent AEs experienced.

Consumers play a crucial role in identifying AEs and provide further understanding of their incidence. Consumer AE reporting processes may differ across countries, where not all have a formal system in place. ${ }^{2}$ In many countries, consumer reporting can be completed over the telephone or via a paper or an electronic form. ${ }^{2,3}$ In Australia, consumers can report AEs directly using an online form on the Therapeutic
Faculty of Pharmacy, Pharmacy and Bank Building A15, The University of Sydney, Science Road, Camperdown, NSW 2006, Australia

Tel +6I 29036654 I

Fax +6I 2 935। 439।

Email parisa.aslani@sydney.edu.au 
Goods Administration (TGA) web site or to their health care professional (HCP), who can report AEs either directly to the TGA, via a web site (Australian Adverse Drug Reaction Reporting System ${ }^{4}$ ), or by telephone, post, e-mail, or fax. ${ }^{5}$ Consumers can also report AEs via the NPS MedicineWise Adverse Medicine Events (AME) Line. ${ }^{6}$ This is a longstanding, free service that enables consumers to report a suspected event, assisted by a pharmacist. The AME Line then reports the suspected AEs to the TGA.

Consumer AE reporting can support signal detection ${ }^{7}$ and lead to the identification of new AEs as well as those previously unreported by HCPs that may potentially be associated with a medicine or vaccine. ${ }^{8}$ To enable effective and timely AE reporting, consumers must have an understanding of what comprises an $\mathrm{AE}$, the motivation to report, and, importantly, an awareness of available reporting systems.

In general, consumer awareness of AE reporting systems is low. ${ }^{9-12}$ Direct consumer reporting of AEs reflects this, with only a small proportion actively reporting to regulatory bodies. An overview of AE reporting in 50 countries found that 44 of these countries have a consumer reporting system that generated a mean of only $9 \%$ of the total AE reports received in 2012. ${ }^{13}$ In 2014 in Australia, only 3\% of AE reports for medicines and vaccines were received from consumers; ${ }^{14}$ for vaccine-related AE reports, the percentage was slightly higher at $4 \%$ in $2014 .{ }^{15}$

There has been an increasing interest in consumer reporting of AEs. Consumers feel positively toward a reporting system that does not rely on HCP reporting. ${ }^{12,16,17}$ Facilitators for consumer AE reporting include the desire to provide insight into their own experiences, ${ }^{17,18}$ help others avoid the AEs; ${ }^{12,16,19}$ improve medication safety, awareness, and/or practices; ${ }^{12,16,19}$ the perceived severity of the AEs $;{ }^{18-20}$ the desire for further information about AEs; ${ }^{16,19}$ and prompting of self-reports either by others or due to HCPs not actively reporting them. ${ }^{16,19}$ Conversely, not all consumers perceive that the onus for AE reporting lies with them; it was seen as the doctor's responsibility. ${ }^{11,12}$ Other key barriers to AE reporting include consumer perceptions that the AE was anticipated or lacked severity, ${ }^{20}$ a lack of consumer reporting system awareness, ${ }^{19}$ issues associated with the reporting process, ${ }^{19}$ and a lack of perceived benefit associated with AE reporting due to expected symptom resolution. ${ }^{19}$

When comparing the number of medicine- and vaccinerelated AEs reported by consumers to the TGA ${ }^{14}$ in previous studies, where the proportion who had experienced a side effect
(SE) ranged between $\sim 17 \%-25 \%{ }^{10,17,21}$ and $46 \%-58 \%,{ }^{9,20,22}$ there is a significant discrepancy between direct consumer $\mathrm{AE}$ reports received and actual $\mathrm{AE}$ incidence. Direct reporting by consumers to the TGA has been available for over 50 years ${ }^{3}$ and yet remains an underutilized option.

Previous research has been conducted with consumers on reporting of AEs in other countries; ${ }^{11,12,16,17,23-25}$ however, there is little published qualitative research on the opinions of Australian consumers on the risks associated with medicines or their role in reporting AEs. Thus, this study aimed to explore:

1) consumer opinions about AEs potentially associated with medicines and vaccines; and

2) consumer views on, and experiences with, managing and reporting AEs.

\section{Materials and methods}

A qualitative study was undertaken using focus groups to address the study aims. Focus groups facilitate the simultaneous gathering of several individuals' views ${ }^{26}$ and allow valuable insights to be gained into issues potentially less accessible without the synergy from group discussion. ${ }^{27}$ The study followed and has been reported according to the consolidated criteria for reporting qualitative research. ${ }^{28}$

\section{Recruitment and sample size}

Convenience sampling was used to encompass a spectrum of people (and their carers) who used medicines and vaccines, were of various ages, and with varying ethnic and socioeconomic backgrounds. Recruitment was conducted by a market research company. Potential participants were screened and, if eligible and consenting, were provided with further study information. People were eligible if they were:

- at least 18 years old;

- able to participate without a translator;

- were taking (or had taken in the past 12 months) at least one prescription medicine; or

- were a parent/carer of a child who had received a vaccination in the past 5 years.

Each participant was reimbursed AUD\$85 for their time and travel expenses.

\section{Focus group conduct}

Focus groups consisting of 6-8 participants were held at various locations across metropolitan Sydney (March-April 2014). Upon arrival, the participants were provided with 
the Participant Information Statement to read and a written consent form and demographics questionnaire to complete. All discussions were facilitated by an experienced female facilitator $(\mathrm{KH})$. A second female researcher was also present to assist with the facilitation and to take field notes (PA). Each focus group lasted $\sim 1.5$ hours and was audio-recorded with the permission of the participants.

A semi-structured topic guide was used to facilitate the discussions and elicit consumers' experiences and opinions about AEs associated with medicines and vaccines, with a focus on:

1) consumers' understanding of, and terminology associated with, AEs;

2) consumers' opinions and experiences of AEs;

3) consumers' opinions about the balance between the benefits and harms in the management or prevention of their health problems (associated with their medicines and vaccines); and

4) actions taken by consumers when experiencing AEs.

The term "SEs" was used during the focus group discussions as it is regarded as an older and more established term for consumers and HCPs when referring to an AE due to a medicinal product or other therapeutic goods, and was known to all participants. Furthermore, Australian Consumer Medicine Information (CMI) leaflets utilize the term SEs rather than terms such as adverse drug reaction or $\mathrm{AE}$.

\section{Data analysis}

Audio-recordings were transcribed verbatim and thematically analyzed using techniques from the grounded theory method. ${ }^{29}$ Transcripts were reviewed and analyzed by a researcher $(\mathrm{KH})$ who coded, categorized, and summarized the data; another researcher (PA) independently cross-checked and reviewed them for consistency. Comments were also received from a research team member who attended two focus groups as an observer (TM).

The coding categories organized the content according to the major overarching themes derived from the protocol, and within these, several subthemes emerged. As themes and subthemes developed, a system of "constant comparison," through the cross-referencing of emerging and recognized themes, was used. Consequently, the data were recoded periodically, either merging into larger themes or declustering into more specific subthemes. Ongoing discussion and review between the researchers and further refining of the coding frames reconciled any discrepancies. Theoretical data saturation ${ }^{30}$ was reached at the fifth focus group and confirmed in the sixth focus group.

\section{Compliance with ethical standards} Ethical approval and informed consent

The study received approval from the Human Research Ethics Committee of The University of Sydney. The participants provided written informed consent. All procedures performed in studies involving human participants were in accordance with the ethical standards of the University of Sydney.

\section{Results}

\section{Participant characteristics}

Forty-eight participants were recruited for six focus groups. There was an equal proportion of men and women, with a median age of 42.5 years (range, 19-73 years). Over a third of the sample had a university education (Table 1). About half of the participants reported that they had experienced an $\mathrm{AE}$ in the past. This may have been due to a medicine or vaccine or both.

Five broad themes were derived from the data:

1) participants' understanding of the term SE and likelihood and severity of SEs;

2) balancing harms versus benefits of medicines;

3) sources of information on SEs;

4) experience with SEs; and

5) reporting of SEs.

Table I Summary of participant demographics

\begin{tabular}{ll}
\hline Characteristics & $\mathbf{n}(\%)(\mathbf{n}=\mathbf{4 8})$ \\
\hline Gender & \\
Female & $24(50)$ \\
Male & $24(50)$ \\
Country of birth & \\
$\quad$ Australia & $38(79.2)$ \\
Other & $10(20.8)$ \\
Language spoken at home & \\
English & $46(95.8)$ \\
Other & $2(4.2)$ \\
Level of education & \\
Year $\leq$ I2 & $19(39.6)$ \\
Certificate/diploma/TAFE/trade certificate & $8(16.7)$ \\
Undergraduate & $17(35.4)$ \\
Postgraduate & $2(4.2)$ \\
Carer status/role & \\
Parent & $23(47.9)$ \\
Carer of an adult & $9(I 8.8)$ \\
Has a carer & $3(6.3)$ \\
Experienced an AE & \\
Medicine & $21(43.8)$ \\
Vaccine & $27(56.3)$ \\
Number of medicines (mean per person) & 4.3 \\
\hline Note: &
\end{tabular}

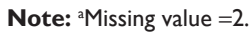

Abbreviations: AE, adverse event; TAFE, Technical And Further Education. 
When discussing medicines, the participants referred to prescribed medicines and not over-the-counter (OTC) or complementary and alternative medicines (CAMs). They considered it unlikely that they would experience SEs with CAMs or OTC medicines, and they did not report any major problems or issues. OTC medicines were considered less of a risk and "safer" than prescription medicines, but still not without potential for side effects or drug interactions.

\section{Understanding of the term "SE," likelihood, and severity}

When the term "SEs" was discussed, the participants commonly regarded them as "out of the ordinary," "negative," "unwanted," "unintentional," "an overreaction," or an "allergic reaction." A few mentioned SEs could be both "positive and negative," and one person said they could be "emotional, even psychological." Some participants considered SEs as something you "will experience quite soon after taking the medication" or "after a period of time."

Although the participants were referring to SEs as reactions, only a few were aware of alternative terms such as "adverse effects," "reactions," "symptoms," or "risks." The knowledge of these terms was derived from either the medicine label, leaflet, or online when searching for information on SEs.

Most participants did not expect to experience SEs. A few believed that doctors would prescribe appropriate medicines that were suitable and were less likely to cause SEs. The overall belief was that medicines have SEs, and in some cases, SEs will definitely happen.

[A] medicine is something that is meant to flush out some things inside your body. But because you do, it attacks everything else as well. So that is why you get the side effects, in my opinion. So you will most likely always get some kind of side effects from most medicines. [Focus group (FG) 1, male (M) 3]

Well, I would accept that if it's common, it's going to

happen sooner or later. [FG1, Female (F) 3]

If it's common, I think it would happen to me. If it's rare, like it won't happen to me as I feel healthy. [FG1, F2]

When questioned about the likelihood of experiencing a "common" SE, individual participant estimates ranged from $<10 \%$ to $75 \%$. One participant thought that the likelihood had to be $<10 \%$, while a few others considered the range to be $10 \%$ or $20 \%$. Participants justified this by stating that if the incidence was higher, the medicine would not be available to the general public. Overall, and after the discussion, the general consensus among the participants was at least $50 \%$ and up to $75 \%$.

I would only put it as $20 \%$. If I am taking medicine, I don't expect to get a side effect. I think if you get too many side effects, I'm shocked that the medication has been approved. ... I don't take medication assuming that there would be a side effect. [FG6, F1]

Participants were asked to quantify the incidence of "rare" SEs. There was a general consensus that the likelihood of rare SE occurrence was $<10 \%$ with a variation in responses from " $<1 \%$ " to "single digits."

Participants largely considered that SE severity should not be worse than the symptoms they were already treating. Based on this, consumers' acceptance of severity of SEs was mild.

I don't think it's going to be severe but if it is, it's a sure sign that you shouldn't be on that medication. ... I don't believe in that you've got to get sicker before you get better - that is not the idea of taking medication. [FG6, M1]

The perception of severity was also influenced by their experiences or if they have or had a condition which may relate to the SE. For example, if the consumer suffered from migraines, their perception of a listed SE such as "headache" would likely be more severe for them. They were also aware that reactions to, and experiences with, medicines could vary considerably as "everybody is different and not everybody will have the same reaction." [FG3, M3]

\section{Balancing harms versus benefits of medicines}

Factors influencing the balance of harm versus benefit The assessment of harms versus benefits was influenced by a number of factors. The benefit of the medicine was intrinsic, almost assumed, and not consciously thought of by participants when a medicine was prescribed. SEs, however, were something people were conscious of to varying degrees. The "tipping point" for the harm outweighing the benefit was multifactorial. Most agreed it would be different for each person, depending on the individual's circumstances, health, disease state, age, experiences, and beliefs.

Individuals' consideration and expectation of harms versus benefits of medicines varied. Some participants were philosophical about their expectations, others were influenced by simply wanting to feel better, and some based their perception on how necessary a medicine was for their life or health. The first group was grounded in their faith in the regulated 
system for medicines, even though the vast majority were unaware of the regulations governing medicines in Australia. They believed that medicines will not be available if their SEs outweighed their benefits. While the second group was driven to feel better, they did not consider SEs to be relevant. To these individuals, the benefits of medicines far outweighed any potential SEs.

Those who considered their assessment of harms and benefits on their health to ultimately involve choosing between two bad options perceived that their current state of health had to be at least worse than the potential SEs of the medicines. Therefore, they were willing to take the medicine to improve their current condition.

I think there's a major difference between the chemotherapy and the [codeine-containing analgesic] between controlling the pain and curing the disease ... I think chemotherapy cures the disease whereas the [codeine-containing analgesic] just controls the pain. So I think one is indispensable and the other is non-obligatory. [FG1, M4]

There was a general consensus among the group that the condition being treated influenced their perceptions of the likelihood and severity of SEs. If the situation involved life or death scenarios, such as cancer treatment or infertility, they would put up with any sort of SE, no matter how adverse to achieve a positive outcome. Knowing in advance what to anticipate regarding potential serious and debilitating SEs was beneficial to some to manage their expectations.

... it depends on what you are treating. Serious things like cancer, you know you're going to need a really powerful drug to help kill the cancer so you're going to expect certain side effects. But if you're taking something for a headache, you know a mild headache, you shouldn't expect to receive anything. It's a kind of benefit weigh up. [FG6, F1]

Mostly, the harm versus benefit balance depended on the severity of the SEs, participants' own tolerance level to the particular SE, and whether this affected their day-to-day living and quality of life. The majority of participants said that they would tolerate having SEs if the medicine was to be taken short term and would alleviate their condition. Participants indicated that they would be more likely to put up with the discomfort of SEs, even if more severe, if their condition caused them pain and the medicine would lessen the pain. Some participants spoke of how they tolerated SEs because of the potential prevention of harm. For example, if the medication reduced the pain for the migraine sufferer, they would be more willing to tolerate a less severe headache as an SE while other individuals related short-term constipation or diarrhea as being acceptable if the medication alleviated irritable bowel syndrome.

\section{Moderating expectations of SEs}

The participants had an expectation or a sense of inevitability of developing SEs associated with some medicines, particularly vaccinations. Parents stated that their perceptions surrounding SEs and potential for harm were very different when they were considering a medicine for their child compared with themselves. Risks were more of a consideration when making decisions about vaccinations, and they were genuinely more concerned about reactions. However, they were motivated by the greater potential benefit for their children and other children, despite knowing about the SEs or having observed the SEs in friends or relatives. These individuals were more likely to read information or listen to the media about medication SEs. As a result, they were more watchful for SEs and surprised when the anticipated SE was not experienced, especially as the media portrayed the SE as inevitable.

If it was for my daughter, I would probably be right on to it - go straight back to the doctors and look into it, because I would be quite concerned. With myself, I would probably be a lot more relaxed. [FG6, F1]

\section{Information sources for SEs}

Doctors and, to a lesser extent, pharmacists played a vital role in consumers' overall experiences from prescribing to receiving information about a condition, disease, and medicines. Participants trusted their doctors to prescribe medicines that would not cause harm and were right for their condition. They looked to the doctor to also provide information on the medicine, while others trusted pharmacists more in providing this information as they were believed to be more knowledgeable and accessible. When there is a need for information, the doctor or pharmacist was considered a vital initial source of information. To a lesser extent, individuals would also speak to friends, family members, and nurses to gather further information. Pharmaceutical manufacturers were not considered as a useful source of information.

The information received from the doctor or pharmacist about a medicine was taken into consideration when deciding on using the medicine; however, most people stated that they were not completely influenced by reading this information. Participants were aware that culture, age, literacy, and underlying health beliefs about medicines influenced how 
individuals sought information from the Internet or read about SEs in medicine leaflets. This was evident as three distinct groupings among the participants developed as to when they preferred to research and read about the medication:

1) the "early seekers" of information;

2) the "as it happens" users of information; and

3) the "trusting/accepting" of information.

The "early seekers" of information liked to read or research to enhance their understanding about SEs prior to taking the medication or early in the course of therapy. The "as-it-happens" group stated that they would not read or research information regarding SEs until they were experiencing an issue. The third grouping was the "trusting/ accepting" who simply trusted the doctor's judgment despite wanting to be informed. Interestingly, several participants in the "early seekers" of information group admitted that once they read about an SE, they waited and expected to experience an SE.

Overall, the response to reading about SEs and its influence, whether positive or negative, on medicine-taking behavior was mixed. Some participants indicated that they would continue to take their medicine and, if concerned, perform further research or else speak with their doctor or pharmacist.

To obtain further information, almost all participants regardless of age-group used what they referred to as "Dr Google" (the Internet) to search for information about their medicines, condition, or disease.

There's no doubt the Internet now has given people a lot more access to information about what medications they're taking. Whereas before, 20 years ago, you relied on your doctor ... Whereas now, people can actually research potential side effects on the Internet ... [FG6, M1]

One participant spoke of being empowered by using the Internet as a tool to gain information. This allowed them to become better informed prior to the doctor's visit so they were confident and better able to have an informed conversation, leading to appropriate questions and subsequent decisions on treatment. The other participants agreed, although they were aware and mindful of the limitations of the Internet. One limitation raised was a lack of a set search strategy, where participants tended to "Google" the medicine's name along with the term SEs. Even though the Internet was often used for gaining information, there was a healthy skepticism of the credibility of the information found. Information credibility was determined through whether other web sites said the same thing or by relying on their doctor or pharmacist to assist in the interpretation, assessment, and relevance of the information to their condition.

Most participants stated that they tended to use a variety of web sites including university, hospital, and government web sites. University web sites were described as independent and more reliable. Manufacturers' web sites were occasionally used; however, overall participants were skeptical of the information and felt that it was biased.

I think there's many ways. The first way is to obviously ask the GP [general practitioner] and the second way is to check the pamphlet. The third way is Google, but also, if you know someone who has some experience and the doctor, you feel you can ask them ... I would always ... contrary to what you said, I would always go to another GP if I'm not sure because you want a second opinion. [FG1, M3]

Interestingly, many of the participants read information on Internet forums, although no one admitted to posting on them. Many participants related to others on the forum and looked to them to find out about a suspected SE. These participants felt comforted that others had experienced the same situation, giving them a sense of reassurance, as well as information about what to expect, time to resolution, and health outcomes for the situation.

Often forums, like, a lot of people like to talk about it. I don't believe everything I read. I, sort of, try and gather a bit of information. Also, the companies will often have their product sheets and things like that on there so I'll read them as well. But I do find that forums, usually, if you're going through the same side effects and saying "Well, I've got them as well" and then it, sort of, gives you an indication of how long the side effects will last. So there's not really much you can do about it but at least you, sort of, know that, "Oh maybe in a couple of weeks, it will wear off" or something. [FG5, F4]

\section{Experience of SEs}

How participants dealt with an SE and advice they sought often depended on whether or not the medicine was to be taken in the short or long term. Even though experiences were varied, there generally was a similar path of determining whether they were experiencing an SE. Participants would often try discontinuing the medicine on their own and then restarting and seeking further information either through the Internet, the CMI leaflet, or family/friends, and if the issue was severe enough, they would visit their doctor. If they visited the doctor, generally another medicine was prescribed and the doctor recorded the SE information in their file. 
Several participants acknowledged that trying to determine whether the SE was related to their medicine or a byproduct of their condition was not always straightforward and often proved difficult. Eventually, they would seek further information or go to their doctor, who sometimes was not able to provide certainty either. Others placed their trust in the doctor and saw it as the doctor's role to use their expertise to determine whether it was an SE.

You need to get confirmation from the doctor that it is a side effect. And let the doctor deal with that one. It's not for me to decide whether it's a side effect or not, even if I think so. [FG4, M2]

Participants with chronic conditions revealed that they would report the SEs first to their doctor and then inquire about an alternative treatment.

If that was me, I would see if it's [the medical condition] life threatening first. And then if it was life threatening, then I would go on it [the medicine] for a while. And then while I was on it, if I knew there was long term side effects, I would be researching at the same time what the other options were. [FG2, F3]

\section{Reporting of SEs}

When the discussion focused on medicine regulation within Australia, the majority had never heard of the TGA and they were unaware of its activities and role. Interestingly, some knew of the US Food and Drug Administration. Regarding the process for a medicine to reach the market, some understood that there were "trials" involving animals and then humans, although they were unaware of the testing process. Essentially, participants assumed that when a medicine was approved, it was "safe." They were completely unaware of where or why they would report SEs for a prescribed or purchased marketed therapeutic product to anyone other than their doctor or even that there are other options available to consumers.

There was an assumption that once an SE was reported to the doctor, whatever was recorded in their file within the doctor's system went into a centralized database accessible by the government, but not by the pharmaceutical manufacturer. There was a group that presumed their doctor reported it to other doctors and had discussions around the issue when they went to conferences. In general, participants mentioned that once their SE was discussed with their doctor, no further consideration was given to the reporting process or what happened to the reported information.
Participants reported that there should be a process for direct consumer SE reporting. However, most said in reality they would be unlikely to report minor SEs and would only report SEs that they considered severe. Interestingly, several participants thought the average "Joe Blow" would not report any SEs, as there was nothing in it for them. Mostly, participants said that they would consider reporting SEs based on whether they had the time and considered them important to report. Participants recognized the value in reporting, not only for themselves but also for the broader community, to improve postmarketing medication safety, monitoring, and vigilance.

Ultimately I think it really helps other people. If it's not going to help you, you already have the symptoms and you can already make a decision: are you going to stop the medication or keep going with it? But it really helps others so that they know you've had these symptoms. [FG5, F4]

However, there were a small number who had no appreciation of the value this information provided to either themselves or to future users of the medicine, until the issue was discussed by other participants.

\section{Preferred reporting system characteristics and processes}

Participants stated that any reporting system implemented had to be easily accessible, quick, and simple to use; otherwise people would not use it. While the Internet was the most suggested system, there was awareness that not all people had access to the Internet and concerns were raised about how well the system would be maintained. Therefore, there was consensus for an alternative method allowing universal access.

There was a general lack of awareness of the existing method of directly reporting SEs to the product manufacturer. A minority of consumers suggested this process. Individuals who did not agree with direct reporting to the manufacturer raised concerns about what would be done with the information as one participant stated that the pharmaceutical manufacturers may "suppress" the information provided about SEs or manipulate it to their benefit. The predominant opinion was that SEs should be reported to the doctor or pharmacist who can determine the genuineness of the claim and whether it was a legitimate SE or an unrelated event. The HCPs would then be responsible and required to report the information to a centralized independent body. This independent group would collate and filter the information, determine whether the reported issues are SEs, and respond to the reporting. Overall, participants preferred this independent body to be a 
government group; however, some expressed concern about the government's efficiency and capacity to do the job in a simple, user-friendly manner.

There was a general agreement that auditing, accountability, and transparency of data were vital for any SE reporting process. Participants expressed the need for tracking exactly what happened to their report, including the report outcome. An e-mail response that provided the assessment of their report and determined whether it was actually an SE or an unrelated event was also considered.

Issues raised by the participants with direct consumer reporting of SEs included corporate espionage, disgruntled people venting on web sites, hypochondriacs, and general vexatious or malicious reporting. Individuals were concerned that these issues would put "noise in the data."

In relation to what information should be supplied in a report, participants suggested basic details; including age, gender, address, contact details; medication details, such as, drug name, strength, how often medication is taken, route of administration; other medications being used at the time of reaction; and postcode. Participants considered the inclusion of postcode to determine clusters in experiencing SEs that may be attributable to other factors. A minority considered reporting should be anonymous, while the majority thought that personal information should be required. The rationale provided was to assist in assessing the relativity of SEs to certain groups of people and to validate the genuineness of the claim.

\section{Discussion}

The term "SE" was most commonly known among the study participants. Assessing harms versus benefits was multifactorial, based on disease state, experiences, and beliefs. Reading about SEs did not alter medicine-taking decisions but made most consumers more alert, aware, and vigilant about SEs. Most had experienced an SE and indicated that they reported SEs to the doctor or pharmacist only if they were severe or bothersome. Participants were generally unaware of AE reporting avenues but saw value in reporting for themselves and the greater medicine-taking population to improve medication safety, monitoring, and quality. Reporting had to be easy, accessible, and time-efficient and participants preferred it to be handled by an independent body, with their doctor as the preferred reporting intermediary. The availability of reported data and transparency of information were deemed important.

An understanding of how consumers identify, appraise, and manage AEs is imperative for furthering pharmacovigilance among medicine users. Similarly, other studies also found that perceptions of the severity of the AEs were also a key driver for AE reporting by consumers. ${ }^{18-20}$ With a wide spectrum of SE incidence estimates associated with descriptors such as "common" and "rare," how SE risk information is communicated to, and sought by, consumers may influence expectations about AEs and subsequent reporting. This can be seen in relation to the "early seekers" of information who expected to experience an SE after reading the information. It is well established that the use of verbal descriptors leads to risk overestimation, ${ }^{31}$ which was evident in the present study. In Australia, numerical SE risk information is not commonly provided in CMI leaflets; however, verbal descriptors are used. Thus, writers of medicine information must be cognizant of the limitations of present communication strategies. Concerted efforts are needed to improve consumer understanding of SE information.

Three consumer profiles were previously identified, ${ }^{32}$ linked to the evaluation of written SE risk information and perceived individual risk: those who felt more at a risk of experiencing an SE; those who felt had less individual risk; and those who were unsure of their own individual risk. Consumers who viewed themselves as more sensitive to medicines have been associated with an increased likelihood of medicine information seeking as well as a higher number of self-reported symptoms experienced. ${ }^{33}$ Parrella et a ${ }^{34}$ also found that when comparing between $\mathrm{AE}$ reporters and non-AE reporters, reporters more likely perceived their child to have been at a risk of experiencing a serious $\mathrm{AE}$ linked to the most recent immunization. Thus, consumer SE risk perception profiles, ${ }^{32}$ together with the three groups identified in the present study (the "early seekers" of information, "as it happens" information users, and those "trusting/accepting" of information), can provide further insight into consumer reporting and strategies to optimize SE information communication. A range of strategies such as focusing on how consumers seek information and interpret risk information may be of benefit.

Considering that the number of reports received directly from consumers by the TGA has not exceeded 5\% over the past few years, ${ }^{14,35-37}$ there appears to be minimal consumer engagement in reporting SEs to the TGA. While a direct method of reporting was preferable for some, the predominant opinion was that SEs should be reported to the doctor (or the pharmacist, as suggested by some) who would then filter and report this information. This may be due to consumers' perceptions that they may need assistance in determining whether they are experiencing an SE. Reasons for reporting an SE are to stop the SE (if severe and undesirable) by 
changing the medicine or to seek reassurance and continue with the medicine if the benefit outweighs the harm. This can only be achieved when reporting directly to an HCP rather than to the TGA or manufacturer. Moreover, direct consumer reporting to regulatory bodies such as the TGA should not replace direct consumer reporting to the prescribing doctor or dispensing pharmacist, as these HCPs are key in interpreting the $\mathrm{AE}$ and taking action to assist the consumer. While HCPs should be responsible for reporting an AE to the TGA, this can be done in partnership with the consumer involved, and vice versa. Key benefits of this approach would be accurate and timely submission of AE reports, without duplication, and ongoing education of the consumer about the reporting process.

The lack of consumer awareness of AE reporting systems is consistent with the literature. ${ }^{19,22,38}$ Participants' concerns surrounding consumer reporting of AEs demonstrated a lack of understanding of TGA reporting processes for AEs and their ongoing analysis to identify potential problems for further investigation. Widespread lack of awareness contributes to the lack of direct AE reports from consumers. This, together with existing consumer knowledge gaps and misunderstandings regarding AE reporting, needs to be addressed. Public health initiatives and consumer education strategies could focus on educating consumers on recognizing AEs, how and where to report them, and the importance and value of $\mathrm{AE}$ reporting for patient safety.

This study has some limitations. Being a qualitative study, with focus groups conducted in the Sydney metropolitan area, the findings are not generalizable to the broader population. In addition, sampling bias may also be evident toward those who may be interested in the study and who had experienced SEs.

\section{Conclusion}

Most participants did not expect to get an SE despite being aware of their potential. Incidence estimates for "common" and "rare" SEs were high and variable. Many were unaware of consumer AE reporting schemes. Participants said that they would like SEs to be reported to an independent or government body, and many expressed that they would report to a doctor or other HCP as an intermediary. Consumers would report SEs generally if serious, although they were unlikely to report them if it did not impact their daily life. Consumers saw the value of reporting suspected SEs not only to benefit themselves but the wider medicine/vaccine user community. The key finding of lack of awareness of consumer reporting mechanisms and the TGA's regulatory function, as well as the preference to report to an HCP (namely their doctor), points to the need to raise awareness of consumers about AEs, including the value of such reports. Continued research may further assist in understanding both consumer and HCP motivations when reporting AEs and establishing more accessible methods of reporting for both groups in order to support their needs and preferences.

\section{Acknowledgments}

The authors would like to thank the participating consumers who attended the focus group discussions. The authors acknowledge ARCS stands for the Association of Regulatory and Clinical Scientists (ARCS) Australia for administrative project support. This research study received funding from the Australian Government TGA.

\section{Disclosure}

$\mathrm{KH}$ is currently employed by CaPPRe P/L, a company that provides independent research services to the government and pharmaceutical industry. JC is employed by the Australian Government TGA, who provided funding for the study. At the time of the study, CM was employed by the Consumers Health Forum, which has an advocacy interest in ensuring that consumers are well informed about AEs. The authors report no other conflicts of interest in this work.

\section{References}

1. World Health Organization. Safety Monitoring of Medicinal Products: Reporting System for the General Public. Geneva: World Health Organization; 2012.

2. Matos C, Härmark L, van Hunsel F. Patient reporting of adverse drug reactions: an international survey of national competent authorities' views and needs. Drug Saf. 2016;39:1105-1116.

3. van Hunsel F, Härmark L, Pal S, Olsson S, van Grootheest K. Experiences with adverse drug reaction reporting by patients: an 11-country survey. Drug Saf. 2012;35:45-60.

4. TGA eBusiness Services. Australian Adverse Drug Reaction Reporting System. Available from: https://www.ebs.tga.gov.au/ebs/ADRS/ ADRSRepo.nsf?OpenDatabase. Accessed February 27, 2017.

5. Australian Government Department of Health Therapeutic Goods Administration. Reporting adverse drug reactions: information for health professionals; 2014. Available from: https://www.tga.gov.au/publication/ reporting-adverse-drug-reactions. Accessed February 27, 2017.

6. NPS MedicineWise. Adverse Medicine Events Line. Available from: https://www.nps.org.au/adverse-medicine-events-line. Accessed February 22, 2018.

7. Härmark L, van Hunsel F, Grundmark B. ADR reporting by the general public: lessons learnt from the Dutch and Swedish systems. Drug Saf. 2015;38:337-347.

8. McGuire T, Moses G. What do consumers contribute to pharmacovigilance? Lessons from the AME Line. In: Conference Proceedings, National Medicines Symposium; March 29-31, 2006; Canberra, Australia. Available from: http://www.icms.com.au/nms2006/abstract/132.htm. Accessed February 27, 2017.

9. Robertson J, Newby DA. Low awareness of adverse drug reaction reporting systems: a consumer survey. Med J Aust. 2013;199:684-686. 
10. Fortnum H, Lee AJ, Rupnik B, Avery A; on behalf of the Yellow Card Study Collaboration. Survey to assess public awareness of patient reporting of adverse drug reactions in Great Britain. J Clin Pharm Ther. 2012;37:161-165.

11. Lorimer S, Cox A, Langford NJ. A patient's perspective: the impact of adverse drug reactions on patients and their views on reporting. J Clin Pharm Ther. 2012;37:148-152.

12. Arnott J, Hesselgreaves H, Nunn AJ, et al. What can we learn from parents about enhancing participation in pharmacovigilance? Br J Clin Pharmacol. 2013;75:1109-1117.

13. Margraff F, Bertram D. Adverse drug reaction reporting by patients: an overview of fifty countries. Drug Saf. 2014;37:409-419.

14. Australian Government Department of Health Therapeutic Goods Administration. Medicines and vaccines post-market vigilance: statistics for 2014. Canberra: Therapeutic Goods Administration; 2015. Available from: https://www.tga.gov.au/sites/default/files/medicinesand-vaccines-post-market-vigilance-statistics-2014.pdf. Accessed March 5, 2017.

15. Dey A, Wang H, Quinn HE, Hill R, Macartney KK. Surveillance of adverse events following immunisation in Australia annual report, 2014. Commun Dis Intell Q Rep. 2016;40:E377-E390.

16. Anderson C, Krska J, Murphy E, Avery A; on behalf of the Yellow Card Study Collaboration. The importance of direct patient reporting of suspected adverse drug reactions: a patient perspective. $\mathrm{Br}$ J Clin Pharmacol. 2011;72:806-822.

17. Jarernsiripornkul N, Patsuree A, Krska J. Public confidence in ADR identification and their views on ADR reporting: mixed methods study. Eur J Clin Pharmacol. 2017;73:223-231.

18. van Hunsel F, van der Welle C, Passier A, van Puijenbroek E, van Grootheest K. Motives for reporting adverse drug reactions by patient-reporters in the Netherlands. Eur J Clin Pharmacol. 2010;66: 1143-1150.

19. Al Dweik R, Stacey D, Kohen D, Yaya S. Factors affecting patient reporting of adverse drug reactions: a systematic review. $\mathrm{Br} J$ Clin Pharmacol. 2017;83:875-883.

20. Matos C, van Hunsel F, Joaquim J. Are consumers ready to take part in the Pharmacovigilance System? - A Portuguese preliminary study concerning ADR reporting. Eur J Clin Pharmacol. 2015;71:883-890.

21. Narumol J, Arunrot P, Krska J. Survey of patients' experiences and their certainty of suspected adverse drug reactions. Int J Clin Pharm. 2015; 37:168-174.

22. Krska J, Morecroft CW. Patients' use of information about medicine side effects in relation to experiences of suspected adverse drug reactions: a cross-sectional survey in medical in-patients. Drug Saf. 2013;36: 673-680.

23. Chaipichit N, Krska J, Pratipanawatr T, Uchaipichat V, JarernsiripornkulN. A qualitative study to explore how patients identify and assess symptoms as adverse drug reactions. Eur J Clin Pharmacol. 2014;70: 607-615.

24. Rolfes L, Wilkes S, van Hunsel F, van Puijenbroek E, van Grootheest K. Important information regarding reporting of adverse drug reactions: a qualitative study. Int $J$ Pharm Pract. 2014;22:231-233.
25. Krska J, Anderson C, Murphy E, Avery AJ, on behalf of the Yellow Card Study Collaboration. How patient reporters identify adverse drug reactions: a qualitative study of reporting via the UK Yellow Card Scheme. Drug Saf. 2011;34:429-436.

26. Morgan DL, Krueger RA. The Focus Group Kit. Thousand Oaks, CA: Sage Publications; 1998.

27. Rubin HJ, Rubin IS. Qualitative Interviewing: The Art of Hearing Data. Thousand Oaks, CA: Sage Publications; 1995.

28. Tong A, Sainsbury P, Craig J. Consolidated criteria for reporting qualitative research (COREQ): a 32-item checklist for interviews and focus groups. Int J Qual Health Care. 2007;19:349-357.

29. Krueger RA, Casey MA. Focus Groups: A Practical Guide for Applied Research. 4th ed. Thousand Oaks, CA: Sage Publications; 2009.

30. Berg BL. Qualitative Research Methods for the Social Sciences. 6th ed. Boston, MA: Pearson/Allyn \& Bacon; 2007.

31. Buchter RB, Fechtelpeter D, Knelangen M, Ehrlich M, Waltering A. Words or numbers? Communicating risk of adverse effects in written consumer health information: a systematic review and meta-analysis. BMC Med Inform Decis Mak. 2014;14:76.

32. Tong V, Raynor DK, Blalock SJ, Aslani P. Consumer interpretation of ramipril and clopidogrel medication risk information - implications for risk communication strategies. Patient Prefer Adherence. 2015; 9:983-988.

33. Faasse K, Grey A, Horne R, Petrie KJ. High perceived sensitivity to medicines is associated with higher medical care utilisation, increased symptom reporting and greater information-seeking about medication. Pharmacoepidemiol Drug Saf. 2015;24:592-599.

34. Parrella A, Gold M, Braunack-Mayer A, Baghurst P, Marshall H. Consumer reporting of adverse events following immunization (AEFI): identifying predictors of reporting an AEFI. Hum Vaccin Immunother. 2014;10:747-754.

35. Australian Government Department of Health Therapeutic Goods Administration. Australian statistics on medicines and vaccines 2012. Canberra: Therapeutic Goods Administration; 2013. Available from: https://www.tga.gov.au/sites/default/files/medicines-statistics-2012. pdf. Accessed March 8, 2017.

36. Australian Government Department of Health Therapeutic Goods Administration. Medicines and vaccines adverse event reports: statistics for 2013. Canberra: Therapeutic Goods Administration; 2014. Available from: https://www.tga.gov.au/sites/default/files/medicines-statistics2013.pdf. Accessed March 8, 2017.

37. Australian Government Department of Health Therapeutic Goods Administration. Medicines and vaccines post-market vigilance: statistics for 2015. Canberra: Therapeutic Goods Administration; 2016. Available from: https://www.tga.gov.au/sites/default/files/medicinesand-vaccines-post-market-vigilance-statistics-2015.pdf. Accessed March 8, 2017.

38. Sales I, Aljadhey H, Albogami Y, Mahmoud MA. Public awareness and perception toward adverse drug reactions reporting in Riyadh, Saudi Arabia. Saudi Pharm J. 2017;25:868-872.
Patient Preference and Adherence

\section{Publish your work in this journal}

Patient Preference and Adherence is an international, peer-reviewed, open access journal that focuses on the growing importance of patient preference and adherence throughout the therapeutic continuum. Patient satisfaction, acceptability, quality of life, compliance, persistence and their role in developing new therapeutic modalities and compounds to optimize

\section{Dovepress}

clinical outcomes for existing disease states are major areas of interest for the journal. This journal has been accepted for indexing on PubMed Central. The manuscript management system is completely online and includes a very quick and fair peer-review system, which is all easy to use. Visit http://www. dovepress.com/testimonials.php to read real quotes from published authors. 